\title{
MPCC: Strong Stability of M-stationary Points
}

\author{
Harald Günzel $^{1}$ • Daniel Hernández Escobar ${ }^{2}$ • Jan-J. Rückmann²
}

Received: 17 August 2020 / Accepted: 13 May 2021 / Published online: 8 June 2021

(C) The Author(s) 2021

\begin{abstract}
In this paper we study the class of mathematical programs with complementarity constraints MPCC. Under the Linear Independence constraint qualification MPCC-LICQ we state a topological as well as an equivalent algebraic characterization for the strong stability (in the sense of Kojima) of an M-stationary point for MPCC. By allowing perturbations of the describing functions up to second order, the concept of strong stability refers here to the local existence and uniqueness of an M-stationary point for any sufficiently small perturbed problem where this unique solution depends continuously on the perturbation. Finally, some relations to $\mathrm{S}$ - and C-stationarity are briefly discussed.
\end{abstract}

Keywords Mathematical programs with complementarity constraint · M-stationarity · Strong stability · Algebraic characterization · MPCC-LICQ

Mathematics Subject Classification (2010) 90C31 · 90C30 · 90C33 · 49K40

\section{Introduction}

In this paper we consider the following mathematical program with complementarity constraints (MPCC):

$$
\mathcal{P}^{\mathrm{cc}}(f, r, s): \min _{x \in M[r, s]} f(x)
$$

with

$$
M[r, s]=\left\{x \in \mathbb{R}^{n}: \min \left\{r_{m}(x), s_{m}(x)\right\}=0, m \in L\right\},
$$

Daniel Hernández Escobar

Daniel.Hernandez@uib.no

Harald Günzel

guenzel@mathc.rwth-aachen.de

Jan-J. Rückmann

Jan-Joachim.Ruckmann@uib.no

1 Department of Mathematics, RWTH Aachen University, Aachen, Germany

2 Department of Informatics, University of Bergen, Bergen, Norway 
where $L=\{1, \ldots, l\}, l \in \mathbb{N}$ and all describing functions $f: \mathbb{R}^{n} \rightarrow \mathbb{R}$ and $r_{m}, s_{m}: \mathbb{R}^{n} \rightarrow$ $\mathbb{R}, m \in L$, are assumed to be twice continuously differentiable. There are many applications where MPCC is used, we refer e.g. to [23, 28]. The results of this paper refer to the particular structure imposed by the complementarity constraints. In order to keep the presentation as less technical as possible we did not include additional equality and inequality constraints which are usually used in standard nonlinear optimization. However, all results presented here can be straightforwardly extended to this more general case with these standard constraints.

In this paper we deal with the concept of strong stability to an M-stationary point for MPCC under the Linear Independence constraint qualification for MPCC (MPCC-LICQ). Recall that MPCC-LICQ holds at a feasible point if the gradients of the active constraints at this point are linearly independent.

The concept of strong stability of a stationary point for a standard nonlinear program was introduced by Kojima [21]. It refers to the local existence and uniqueness of a stationary point for each sufficiently small locally perturbed problem where perturbations up to second order (function values, first and second derivative) are allowed. Moreover, this unique stationary point of the perturbed problem depends continuously on the underlying perturbation. Obviously, these "well-posedness" features (existence, uniqueness and continuity on perturbation) play an important role in sensitivity analysis and parametric optimization. Besides the topological definition of strong stability, Kojima [21] proved an equivalent algebraic characterization using second order information and applying matrix analysis. This algebraic characterization can be used to decide whether or not a stationary point is strongly stable. We refer to related papers $[20,25,26]$ on properties on strongly stable stationary points.

The goal of this paper is twofold. First, we introduce the strong stability of an M-stationary point for MPCC. Then, under MPCC-LICQ, we prove an equivalent algebraic characterization of this property. Note that since we only consider complementarity constraints, here, the constraint qualifications MPCC-LICQ and MPCC-MFCQ are identic. In general, they are different due to the presence of additional inequality constraints.

It is well-known that there exist several stationarity concepts for MPCC. Among them are A-, B-, C-, M- and S-stationarity; for an overview and discussion we refer to [29]. There exist already results on strongly stable C-stationary points for MPCC $[4-6,17]$ where this particular interest is motivated by the fact that $\mathrm{C}$-stationarity is related to changes of topological properties of the underlying problem $[15,16]$. However, in this paper we choose the stronger concept of M-stationarity which excludes more (but not all) decent directions than the concept of C-stationarity.

We would like to mention some other related papers: there are many references on stationarity concepts, optimality conditions, sensitivity and other properties for MPCC, see e.g. [1, 3, 10, 18, 24, 27]. Moreover, we refer to some papers where solution methods for MPCC are discussed [2, 8, 11, 19, 22].

This paper is organized as follows. Section 2 provides some notations used later on. In Section 3 we recall the concept of strong stability of a stationary point for a standard nonlinear program. Section 4 contains the new results: We state a topological and, under MPCC-LICQ, an equivalent algebraic characterization of a strongly stable M-stationary point for MPCC. Some final remarks are given in Section 5. 


\section{Some Basic Notations}

Most of the presentation of basic notations in this section is taken from [5]. For $w \in \mathbb{R}^{n}$ let $w=\left(w_{1}, \ldots, w_{n}\right)$ and define

$$
\begin{aligned}
& I^{0}(w)=\left\{i \in\{1, \ldots, n\}: w_{i}=0\right\}, \\
& I^{*}(w)=\left\{i \in\{1, \ldots, n\}: w_{i} \neq 0\right\} .
\end{aligned}
$$

For $\bar{x} \in \mathbb{R}^{n}$ and $\delta>0$ the closed Euclidean ball centered at $\bar{x}$ with radius $\delta$ will be denoted by $B(\bar{x}, \delta)$ and the Euclidean sphere centered at $\bar{x}$ with radius $\delta$ by $S(\bar{x}, \delta)$. We abbreviate the sentence " $V$ is a neighborhood of $\bar{x}$ " by letting $\mathcal{V}(\bar{x})$ to be the set of all neighborhoods of $\bar{x}$. This allows us to write the aforementioned sentence as " $V \in \mathcal{V}(\bar{x})$ ".

Let $\mathcal{C}^{k}\left(\mathbb{R}^{n}, \mathbb{R}^{m}\right)$ be the space of $k$-times continuously differentiable mappings with domain $\mathbb{R}^{n}$ and codomain $\mathbb{R}^{m}$. For $f \in \mathcal{C}^{2}\left(\mathbb{R}^{n}, \mathbb{R}\right)$ denote the partial derivative of $f$ at $\bar{x} \in \mathbb{R}^{n}$ with respect to $x_{i}$ by $\frac{\partial f(\bar{x})}{\partial x_{i}}, i=1, \ldots, n$. In addition, $D_{x} f(\bar{x})$ stands for its gradient taken as a row vector and $D_{x}^{2} f(\bar{x})$ for its Hessian at $\bar{x}$.

For the strong stability we need a seminorm for functions. Let $V \in \mathcal{V}(\bar{x})$ and $\bar{F} \in$ $\mathcal{C}^{2}\left(\mathbb{R}^{n}, \mathbb{R}^{m}\right)$. Following [21], denote

$$
\|\bar{F}\|^{V}=\max \left\{\sup _{x \in V} \max _{i}\left\{\left|\bar{F}_{i}(x)\right|\right\}, \sup _{x \in V} \max _{i, j}\left\{\left|\frac{\partial \bar{F}_{i}(x)}{\partial x_{j}}\right|\right\}, \sup _{x \in V} \max _{i, j, k}\left\{\left|\frac{\partial^{2} \bar{F}_{i}(x)}{\partial x_{j} \partial x_{k}}\right|\right\}\right\},
$$

where the indices $i$ and $j, k$ are varying in the sets $\{1, \ldots, m\}$ and $\{1, \ldots, n\}$, respectively.

\section{Strong Stability for Standard Nonlinear Programs}

In this section we recall the concept of a strongly stable stationary point for a standard nonlinear program. This result was introduced by Kojima [21] and we will use it in the next section for the algebraic characterization of the strong stability of an M-stationary point for a mathematical program with complementarity constraint.

Consider a standard nonlinear program with finitely many equality and inequality constraints given as

$$
\mathcal{P}^{\mathrm{sn}}(f, h, g): \min _{x \in M^{\mathrm{sn}}[h, g]} f(x),
$$

where

$$
M^{\mathrm{sn}}[h, g]=\left\{\begin{array}{l|l}
x \in \mathbb{R}^{n} & \begin{array}{l}
h_{i}(x)=0, \quad i \in I, \\
g_{j}(x) \geq 0, \quad j \in J
\end{array}
\end{array}\right\}
$$

with finite index sets $I$ and $J$ as well as functions $f \in \mathcal{C}^{2}\left(\mathbb{R}^{n}, \mathbb{R}\right), h_{i} \in \mathcal{C}^{2}\left(\mathbb{R}^{n}, \mathbb{R}\right), i \in I$ and $g_{j} \in \mathcal{C}^{2}\left(\mathbb{R}^{n}, \mathbb{R}\right), j \in J$. For a feasible point $\bar{x} \in M^{\mathrm{sn}}[h, g]$ let the index set of active inequality constraints be given as

$$
J_{g}^{0}(\bar{x})=\left\{j \in J: g_{j}(\bar{x})=0\right\} .
$$

For two standard nonlinear programs $P^{1}, P^{2}$ we write $P^{1}=P^{2}$ if they are defined by the same functions $f, h_{i}, i \in I$ and $g_{j}, j \in J$. Moreover, if two problems $P^{1}=$ $\mathcal{P}^{\mathrm{sn}}\left(f^{1}, h^{1}, g^{1}\right)$ and $P^{2}=\mathcal{P}^{\mathrm{sn}}\left(f^{2}, h^{2}, g^{2}\right)$ have the same number of equality and inequality constraints, then the subtraction of these problems is defined by the following expression

$$
P^{1}-P^{2}:=\mathcal{P}^{\mathrm{sn}}\left(f^{1}-f^{2}, h^{1}-h^{2}, g^{1}-g^{2}\right) .
$$


Recall that a point $\bar{x} \in \mathbb{R}^{n}$ is called a stationary point for $P=\mathcal{P}^{\mathrm{sn}}(f, h, g)$ if there exist $\lambda_{i} \in \mathbb{R}, i \in I$ and $\mu_{j} \in \mathbb{R}, j \in J$ such that

$$
D_{x} \mathbf{L}^{\mathrm{sn}}(\bar{x}, \lambda, \mu)=\mathbf{0}^{T}, h_{i}(\bar{x})=0, i \in I, \min \left\{\mu_{j}, g_{j}(\bar{x})\right\}=0, j \in J,
$$

where $\mathbf{0}$ denotes the origin (column vector) in $\mathbb{R}^{n}$ and

$$
\mathbf{L}^{\mathrm{sn}}(x, \lambda, \mu)=f(x)-\sum_{i \in I} \lambda_{i} h_{i}(x)-\sum_{j \in J} \mu_{j} g_{j}(x)
$$

is the Lagrange function for $P$. Let the set of stationary points for $P$ be denoted by $\Sigma(P)$ and the set of $(\lambda, \mu)$ satisfying $(3.1)$ by $\mathcal{L}(P, \bar{x})$. Recall also that the Linear Independence constraint qualification (LICQ) is said to hold at $\bar{x} \in M^{\mathrm{sn}}[h, g]$ if the vectors

$$
D_{x} h_{i}(\bar{x}), i \in I, D_{x} g_{j}(\bar{x}), j \in J_{g}^{0}(\bar{x})
$$

are linearly independent. If LICQ holds at a local minimizer $\bar{x}$ for $P$, then $\bar{x}$ is also a stationary point for $P$. Note that we can generically assume that LICQ holds at any feasible point [13].

As already mentioned, the concept of strong stability of a stationary point was introduced by Kojima [21] and it is related to the maximum modulus of the differences between the values of the functions, and their first and second order derivatives. For this concept we use the following seminorm: given $\bar{x} \in \mathbb{R}^{n}, V \in \mathcal{V}(\bar{x})$ and $P=\mathcal{P}^{\mathrm{sn}}(f, h, g)$, we define

$$
\|P\|^{V}=\|(f, h, g)\|^{V},
$$

where the right-hand-side is obtained from (2.1) by choosing $\bar{F}=(f, h, g)$. Then, for $\bar{P}=\mathcal{P}^{\mathrm{sn}}(\bar{f}, \bar{h}, \bar{g})$ and $\delta>0$ define

$$
B^{V}(\bar{P}, \delta)=\left\{P:\|P-\bar{P}\|^{V} \leq \delta\right\},
$$

where $P$ and $\bar{P}$ have the same number of equality and inequality constraints. Now, we recall Kojima's definition of a strongly stable stationary point.

Definition 3.1 [21] Let $\bar{P}=\mathcal{P}^{\mathrm{sn}}(\bar{f}, \bar{h}, \bar{g})$. A point $\bar{x} \in \Sigma(\bar{P})$ is called strongly stable if there exists a real number $\bar{\delta}>0$ such that for all $\delta \in(0, \bar{\delta}]$ there exists a real number $\varepsilon>0$ such that for every $P \in B^{B(\bar{x}, \bar{\delta})}(\bar{P}, \varepsilon)$ it holds that

$$
|\Sigma(P) \cap B(\bar{x}, \bar{\delta})|=|\Sigma(P) \cap B(\bar{x}, \delta)|=1 .
$$

The set of strongly stable stationary points for $\bar{P}$ is denoted by $\Sigma^{S}(\bar{P})$.

Note that in the previous definition strong stability is stated as a topological property which cannot be straightforwardly checked in general. In this regard, Kojima [21] did not only provide the concept of a strongly stable stationary point, but also an equivalent algebraic characterization of it under the well-known Mangasarian-Fromovitz constraint qualification. In the remainder of this section we recall this characterization for the case that LICQ holds at the considered point. For that we assume:

- $\quad \bar{P}=\mathcal{P}^{\mathrm{sn}}(\bar{f}, \bar{h}, \bar{g})$ with Lagrange function $\overline{\mathbf{L}}^{\text {sn }}$ and $\bar{x} \in \Sigma(\bar{P})$.

- $\quad$ LICQ holds at $\bar{x}$.

- $\quad(\bar{\lambda}, \bar{\mu})$ are the uniquely determined Lagrange multipliers, that is, $\mathcal{L}(\bar{P}, \bar{x})=\{(\bar{\lambda}, \bar{\mu})\}$.

- $\quad$ For each index set $\bar{J}$ with

$$
I^{*}(\bar{\mu}) \subset \bar{J} \subset J_{\bar{g}}^{0}(\bar{x})
$$


Let $C(\bar{J}) \in \mathbb{R}^{n \times(n-|I|-|\bar{J}|)}$ be a matrix whose columns form a basis of the space

$$
T_{\bar{x}}(\bar{h}, \bar{g}, \bar{J})=\left\{\begin{array}{l|l}
z \in \mathbb{R}^{n} & \begin{array}{l}
D_{x} \bar{h}_{i}(\bar{x}) z=0, i \in I, \\
D_{x} \bar{g}_{j}(\bar{x}) z=0, j \in \bar{J}
\end{array}
\end{array}\right\} .
$$

The latter means that $C(\bar{J})$ is a so-called basis matrix of $T_{\bar{x}}(\bar{h}, \bar{g}, \bar{J})$. Note that, by Sylvester's law, the number

$$
\text { sign } \operatorname{det}\left(C(\bar{J})^{T} D_{x}^{2} \overline{\mathbf{L}}^{\mathrm{sn}}(\bar{x}, \bar{\lambda}, \bar{\mu}) C(\bar{J})\right)
$$

does not depend on the particular choice of $C(\bar{J})$. Now, we recall Kojima's result.

Proposition 3.1 [21] Assume that LICQ holds at $\bar{x} \in \Sigma^{S}(\bar{P})$. Then, the following two conditions are equivalent.

(i) $\bar{x} \in \Sigma^{S}(\bar{P})$.

(ii) sign $\operatorname{det}\left(C(\bar{J})^{T} D_{x}^{2} \overline{\mathbf{L}}^{s n}(\bar{x}, \bar{\lambda}, \bar{\mu}) C(\bar{J})\right)$ is constant and nonvanishing for all $\bar{J}$ satisfying (3.2).

Remark 3.1 We use the convention that $\operatorname{det}\left(C(\bar{J})^{T} D_{x}^{2} \overline{\mathbf{L}}^{\mathrm{sn}}(\bar{x}, \bar{\lambda}, \bar{\mu}) C(\bar{J})\right)>0$ whenever $T_{\bar{x}}(\bar{h}, \bar{g}, \bar{J})=\{\mathbf{0}\}$.

We mention that under the Linear Independence constraint qualification, Kojima's concept of strong stability is equivalent to Robinson's concept of strong regularity for generalized equations [25]; see e.g. [12]. In the following section we will use the results presented in the current section in the context of mathematical programs with complementarity constraints.

\section{Strong Stability for M-stationary Points}

This section contains our main result which is the algebraic characterization of strong stability of M-stationary points for MPCC. Here, we return to the mathematical program $P=\mathcal{P}^{\mathrm{cc}}(f, r, s)$ defined in (1.1). The equality and subtraction of problems are defined analogously to those in Section 3. Moreover, for $\bar{x} \in M[r, s]$ we define the active index sets:

$$
\begin{aligned}
\bar{I}_{r}(\bar{x}) & =\left\{m \in L: r_{m}(\bar{x})=0\right\}, \\
\bar{I}_{s}(\bar{x}) & =\left\{m \in L: s_{m}(\bar{x})=0\right\}, \\
I_{r}(\bar{x}) & =\left\{m \in L: r_{m}(\bar{x})=0, s_{m}(\bar{x})>0\right\}, \\
I_{s}(\bar{x}) & =\left\{m \in L: r_{m}(\bar{x})>0, s_{m}(\bar{x})=0\right\}, \\
I_{r s}(\bar{x}) & =\left\{m \in L: r_{m}(\bar{x})=0, s_{m}(\bar{x})=0\right\} .
\end{aligned}
$$

We recall the definition of an M-stationary point for $P$.

Definition 4.1 A point $\bar{x} \in M[r, s]$ is called an $M$-stationary point for $P$ if there exists $(\rho, \sigma) \in \mathbb{R}^{2 l}$ such that

$$
\begin{aligned}
& D_{x} \mathbf{L}^{\mathrm{cc}}(\bar{x}, \rho, \sigma)=\mathbf{0}^{T}, \\
& \rho_{m} \cdot r_{m}(\bar{x})=\sigma_{m} \cdot s_{m}(\bar{x})=0, m \in L, \\
& \rho_{m}>0, \sigma_{m}>0 \text { or } \rho_{m} \cdot \sigma_{m}=0, m \in L,
\end{aligned}
$$


where

$$
\mathbf{L}^{\mathrm{cc}}(x, \rho, \sigma)=f(x)-\sum_{m \in L}\left[\rho_{m} r_{m}(x)+\sigma_{m} s_{m}(x)\right]
$$

is the MPCC-Lagrange function for $P$. We denote the set of M-stationary points by $\Sigma^{M}(P)$ and the set of Lagrange vectors $(\rho, \sigma) \in \mathbb{R}^{2 l}$ satisfying (4.1)-(4.3) by $\mathcal{L}^{M}(P, \bar{x})$.

We also recall that the Linear Independence constraint qualification for $P$ (MPCCLICQ) holds at $\bar{x} \in M[r, s]$ if the vectors

$$
D_{x} r_{i}(\bar{x}), i \in \bar{I}_{r}(\bar{x}), D_{x} s_{j}(\bar{x}), j \in \bar{I}_{s}(\bar{x})
$$

are linearly independent. It is well-known that any local minimizer for $P$ at which MPCCLICQ holds is also an M-stationary point for $P$, see e. g. [3, Theorem 3.9].

In the remainder of this paper we assume that:

- $\quad \bar{P}=\mathcal{P}^{\mathrm{cc}}(\bar{f}, \bar{r}, \bar{s})$ is a fixed MPCC with MPCC-Lagrange function $\overline{\mathbf{L}}^{\mathrm{cc}}$ and $\bar{x} \in M[\bar{r}, \bar{s}]$ is the point under consideration.

- $\quad$ MPCC-LICQ holds at $\bar{x}$ for $\bar{P}$.

- If $\bar{x} \in \Sigma^{M}(\bar{P})$, then $(\bar{\rho}, \bar{\sigma})$ is the corresponding uniquely determined Lagrange vector, i.e. $\mathcal{L}^{M}(\bar{P}, \bar{x})=\{(\bar{\rho}, \bar{\sigma})\}$.

For $(\bar{\rho}, \bar{\sigma})$ we define the sets

$$
\begin{aligned}
& I^{\bar{\rho}}=I^{0}(\bar{\rho}) \cap I_{\bar{r} \bar{s}}(\bar{x}), \\
& I^{\bar{\sigma}}=I^{0}(\bar{\sigma}) \cap I_{\bar{r} \bar{s}}(\bar{x}) .
\end{aligned}
$$

In the previous section we recalled the concept of strong stability of a stationary point for standard nonlinear programs. Now, we generalize it to M-stationary points for MPCC. Analogously to Section 3, we define an appropriate seminorm. Given $V \in \mathcal{V}(\bar{x})$ and $P=$ $\mathcal{P}^{\mathrm{cc}}(f, r, s)$, let

$$
\|P\|^{V}=\|(f, r, s)\|^{V},
$$

where the right-hand-side is obtained from (2.1) by choosing $\bar{F}=(f, r, s)$. For $\delta>0$ let

$$
B^{V}(\bar{P}, \delta)=\left\{P:\|P-\bar{P}\|^{V} \leq \delta\right\} .
$$

Denote for a given $V \in \mathcal{V}(\bar{x})$ the set of all neighborhoods of $\bar{P}$ by $\mathcal{W}^{V}(\bar{P})$.

Definition 4.2 A point $\bar{x} \in \Sigma^{M}(\bar{P})$ is called strongly stable if there exists $\bar{\delta}>0$ such that for all $\delta \in(0, \bar{\delta}]$ there exists $\varepsilon>0$ such that for $P \in B^{B(\bar{x}, \bar{\delta})}(\bar{P}, \varepsilon)$ it holds that

$$
\left|\Sigma^{M}(P) \cap B(\bar{x}, \bar{\delta})\right|=\left|\Sigma^{M}(P) \cap B(\bar{x}, \delta)\right|=1 .
$$

The set of strongly stable M-stationary points for $\bar{P}$ is denoted by $\Sigma^{M S}(\bar{P})$.

Note that Definitions 3.1 and 4.2 are analogous. Obviously, the distinctions between them are given by the class of programs and the stationarity concept under consideration. Now, we present two examples which illustrate M-stationary points that are not strongly stable.

Example 4.1 (c.f. [17, Example 2.1]) Let $n=2, \bar{x}=(0,0)^{T}$ and consider the problem $\bar{P}$ given by

$$
\begin{gathered}
\min x_{1}^{2}+x_{2}^{2} \\
\text { s.t. } \min \left\{x_{1}, x_{2}\right\}=0 .
\end{gathered}
$$

Note that $\bar{x}$ is an M-stationary point with $\bar{\rho}=\bar{\sigma}=0$, see Fig. 1a. 


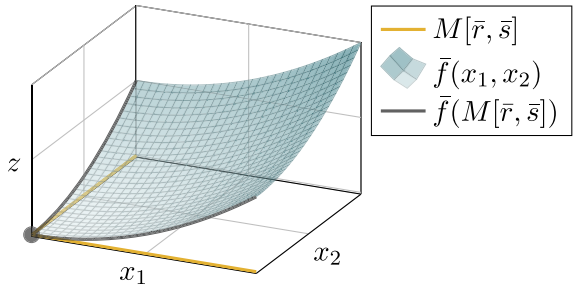

(a) One M-stationary point.

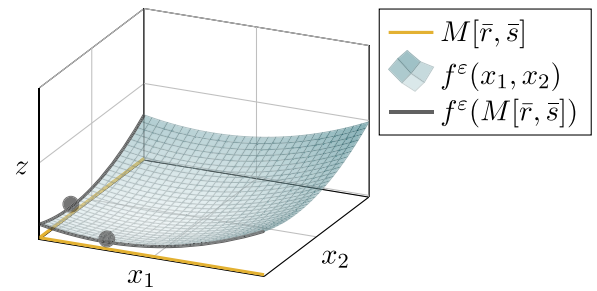

(b) Two M-stationary points.

Fig. 1 Not strongly stable case: two M-stationary points after a sufficiently small perturbation

For $\varepsilon>0$ sufficiently small, consider the perturbation

$$
\begin{aligned}
& \min \left(x_{1}-\varepsilon\right)^{2}+\left(x_{2}-\varepsilon\right)^{2} \\
& \text { s.t. } \min \left\{x_{1}, x_{2}\right\}=0 .
\end{aligned}
$$

It is not hard to see that both $(\varepsilon, 0)^{T}$ and $(0, \varepsilon)^{T}$ are M-stationary points, see Fig. $1 \mathrm{~b}$. Consequently, 0 is not a strongly stable M-stationary point.

Example 4.2 Let $n=2, \bar{x}=(0,0)^{T}$ and consider the problem $\bar{P}$ given by

$$
\begin{gathered}
\min -x_{1}+x_{2}^{2}+1 \\
\text { s.t. } \min \left\{x_{1}, x_{2}\right\}=0 .
\end{gathered}
$$

Note that $\bar{x}$ is an M-stationary point with $\bar{\rho}=-1$ and $\bar{\sigma}=0$, see Fig. 2a.

For $\varepsilon>0$ sufficiently small, consider the perturbation

$$
\begin{aligned}
& \min -x_{1}+\left(x_{2}+\varepsilon\right)^{2}+1 \\
& \text { s.t. } \min \left\{x_{1}, x_{2}\right\}=0 .
\end{aligned}
$$

After a straightforward calculation, it follows that 0 is the only point satisfying (4.2) in Definition 4.1, see Fig. 2b. However, it is not hard to see that 0 is not an M-stationary point for the perturbed problem. Thus, 0 is not a strongly stable M-stationary point for $\bar{P}$.

Examples 4.1 and 4.2 provide some hints for obtaining necessary conditions for strong stability. These will be used when proving the forthcoming Theorem 4.1. Since the proof of the following lemma uses only continuity arguments, we present a short proof of its first statement and skip the proof of the second one.

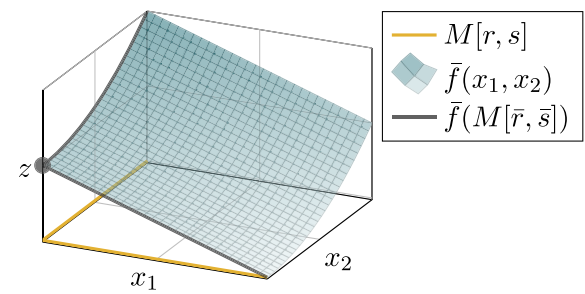

(a) One M-stationary point.

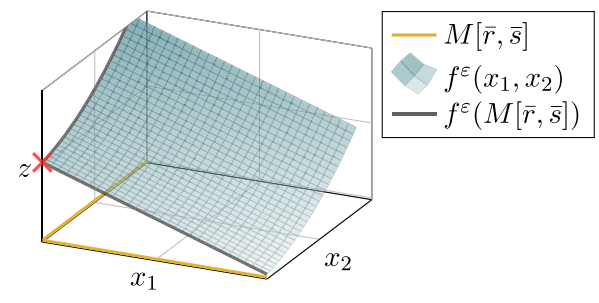

(b) No M-stationary points.

Fig. 2 Not strongly stable case: no M-stationary points after a sufficiently small perturbation 
Lemma 4.1 Assume that MPCC-LICQ holds at $\bar{x} \in \Sigma^{M}(\bar{P})$. Then, there exist $V \in \mathcal{V}(\bar{x})$ and $W \in \mathcal{W}^{V}(\bar{P})$ such that for all $P \in W$ and all $x \in V \cap \Sigma^{M}(P)$ we have that

(1) MPCC-LICQ holds at $x$ for $P$.

(2) The uniquely determined $(\rho, \sigma) \in \mathcal{L}^{M}(P, x)$ satisfies that

- $I^{*}(\bar{\rho}) \subset I^{*}(\rho), I^{*}(\bar{\sigma}) \subset I^{*}(\sigma)$.

- $\rho_{i} \cdot \bar{\rho}_{i}>0, i \in I^{*}(\bar{\rho}), \sigma_{j} \cdot \bar{\sigma}_{j}>0, j \in I^{*}(\bar{\sigma})$.

Proof (1). By a continuity argument on the derivatives of the active constraints, it follows that there exists a compact $V \in \mathcal{V}(\bar{x})$ such that MPCC-LICQ holds for all $x \in V \cap M[\bar{r}, \bar{s}]$. Now, suppose contrarily to (1) that there exist sequences $P^{k}$ with $\left\|P^{k}-\bar{P}\right\|^{V} \rightarrow 0$ and $x^{k} \in V \cap \Sigma^{M}\left(P^{k}\right)$ such that MPCC-LICQ does not hold at $x^{k}$ for $P^{k}$. Hence, for all $k \in \mathbb{N}$ there exists $\left(\alpha^{k}, \beta^{k}\right) \in \mathbb{R}^{2 l}$ with $\left\|\left(\alpha^{k}, \beta^{k}\right)\right\|=1$ such that

$$
\begin{array}{r}
\sum_{m \in L}\left[\alpha_{m}^{k} r_{m}^{k}\left(x^{k}\right)+\beta_{m}^{k} s_{m}^{k}\left(x^{k}\right)\right]=0, \\
\alpha_{m}^{k} \cdot r_{m}^{k}\left(x^{k}\right)=\beta_{m}^{k} \cdot s_{m}^{k}\left(x^{k}\right)=0, m \in L .
\end{array}
$$

By the compactness of $V$ and $\left\|\left(\alpha^{k}, \beta^{k}\right)\right\|=1$, after perhaps reducing to appropriate subsequences, assume that $x^{k} \rightarrow x^{0}$ for some $x^{0} \in V \cap M[\bar{r}, \bar{s}]$ and that $\left(\alpha^{k}, \beta^{k}\right) \rightarrow(\bar{\alpha}, \bar{\beta})$ with $\|(\bar{\alpha}, \bar{\beta})\|=1$. Now, letting $k \rightarrow+\infty$ in (4.4) and (4.5) we get that MPCC-LICQ does not hold at $x^{0}$ and, therefore, a contradiction to the fact that MPCC-LICQ holds for all $x \in V \cap M[\bar{r}, \bar{s}]$.

Next, we present an auxiliary result and a necessary condition for strong stability.

Lemma 4.2 Assume that $\bar{f} \in \mathcal{C}^{\infty}\left(\mathbb{R}^{n}, \mathbb{R}\right)$. If $\bar{f}(0)=0$ and $D_{x} \bar{f}(0)=0$, then there exist $v_{i j} \in \mathcal{C}^{\infty}\left(\mathbb{R}^{1}, \mathbb{R}\right), i, j=1, \ldots, n$ such that

$$
\bar{f}(x)=\sum_{i, j=1}^{n} v_{i j}(x) x_{i} x_{j} .
$$

Proof It follows immediately from Steps 1 and 2 in the proof of [13, Theorem 2.7.2].

Theorem 4.1 Assume that MPCC-LICQ holds at $\bar{x} \in \Sigma^{M S}(\bar{P})$. Then, the following conditions hold:

- $I^{\bar{\rho}} \cap I^{\bar{\sigma}}=\emptyset$.

- $\bar{\rho}_{m} \geq 0, \bar{\sigma}_{m} \geq 0, m \in I_{\bar{r} \bar{s}}(\bar{x})$.

Proof Let $\bar{x} \in \Sigma^{M S}(\bar{P})$ and recall that, consequently, (4.3) in Definition 4.1 holds for $(\rho, \sigma)=(\bar{\rho}, \bar{\sigma})$. Now, suppose contrarily that there exists an index $m^{0} \in I_{\bar{r}}(\bar{x})$ such that one of the following conditions is fulfilled:

- $\bar{\rho}_{m^{0}}=\bar{\sigma}_{m^{0}}=0$.

- $\bar{\rho}_{m^{0}}<0, \bar{\sigma}_{m^{0}}=0$.

- $\bar{\rho}_{m^{0}}=0, \bar{\sigma}_{m^{0}}<0$.

After perhaps adding sufficiently small positive constants to the functions $\bar{r}_{m}, m \in I^{\bar{\rho}} \backslash\left\{m^{0}\right\}$ and $\bar{s}_{m}, m \in I^{\bar{\sigma}} \backslash\left\{m^{0}\right\}$, assume that $I^{\bar{\rho}} \cap I^{\bar{\sigma}}=\left\{m^{0}\right\}$. Next, we will perform several sufficiently small perturbations of $\bar{f}$. Since $\mathcal{C}^{\infty}\left(\mathbb{R}^{n}, \mathbb{R}\right)$ is a dense subset of $\mathcal{C}^{2}\left(\mathbb{R}^{n}, \mathbb{R}\right)[7$, Theorem 2.4], there exists $f^{1} \in \mathcal{C}^{\infty}\left(\mathbb{R}^{n}, \mathbb{R}\right)$ sufficiently close to $\bar{f}$ such that $f^{1}(\bar{x})=\bar{f}(\bar{x})$, 
$D_{x} f^{1}(\bar{x})=D_{x} \bar{f}(\bar{x})$ and $D_{x}^{2} f^{1}(\bar{x})=D_{x}^{2} \bar{f}(\bar{x})$. Analogously, we obtain functions $r^{1} \in$ $\mathcal{C}^{\infty}\left(\mathbb{R}^{n}, \mathbb{R}^{l}\right)$ and $s^{1} \in \mathcal{C}^{\infty}\left(\mathbb{R}^{n}, \mathbb{R}^{l}\right)$ sufficiently close to $\bar{r}$ and $\bar{s}$, respectively. Note that after performing this perturbation the Lagrange vector at $\bar{x}$ for $P^{1}=\mathcal{P}^{\mathrm{cc}}\left(f^{1}, r^{1}, s^{1}\right)$ remains the same.

Moreover, by MPCC-LICQ, for our local consideration we perform a coordinate transformation by choosing as new coordinates a basis of $\mathbb{R}^{n}$ which contains the gradients of the active constraints at $\bar{x}$; see e.g. $[14,16,17]$ where such a coordinate transformation is called standard diffeomorphism. As a consequence, we can restrict ourselves to the following situation: $f^{1} \in \mathcal{C}^{\infty}\left(\mathbb{R}^{2}, \mathbb{R}\right), \bar{x}=(0,0)^{T}, f^{1}(0,0)=0$ and the problem $P^{1}$ becomes

$$
\begin{gathered}
\min f^{1}\left(x_{1}, x_{2}\right) \\
\text { s.t. } \min \left\{x_{1}, x_{2}\right\}=0 .
\end{gathered}
$$

Note that now it holds that

$$
\begin{aligned}
& \frac{\partial f^{1}(0,0)}{\partial x_{1}}=\bar{\rho}=\bar{\rho}_{m^{0}}, \\
& \frac{\partial f^{1}(0,0)}{\partial x_{2}}=\bar{\sigma}=\bar{\sigma}_{m^{0}} .
\end{aligned}
$$

Next, we distinguish two cases.

Case 1: $\bar{\rho}=\bar{\sigma}=0$. A contradiction to this case follows by observing that the two C-stationary points obtained in the proof of Case 2 in [17, Theorem 3.1] are also M-stationary $\mathrm{f}$ completeness we provide here the details of this proof. By Lemma 4.2, we get

$$
f^{1}\left(x_{1}, x_{2}\right)=v_{11}\left(x_{1}, x_{2}\right) x_{1}^{2}+v_{12}\left(x_{1}, x_{2}\right) x_{1} x_{2}+v_{22}\left(x_{1}, x_{2}\right) x_{2}^{2}
$$

for some $v_{11}, v_{12}, v_{22} \in \mathcal{C}^{\infty}\left(\mathbb{R}^{2}, \mathbb{R}\right)$. By possibly adding sufficiently small quadratic terms to $f^{1}$ we assume $v_{11}\left(x_{1}, x_{2}\right) \neq 0$ and $v_{22}\left(x_{1}, x_{2}\right) \neq 0$.

Then, an appropriate local coordinate transformation leaves the feasible set of $P^{1}$ unchanged while the objective function becomes

$$
f^{2}\left(x_{1}, x_{2}\right)=c_{1} x_{1}^{2}+v\left(x_{1}, x_{2}\right) x_{1} x_{2}+c_{2} x_{2}^{2},
$$

where $c_{1}, c_{2} \in \mathbb{R} \backslash\{0\}$ and $v \in \mathcal{C}^{\infty}\left(\mathbb{R}^{2}, \mathbb{R}\right)$. For $\varepsilon>0$ sufficiently small let

$$
f^{\varepsilon}\left(x_{1}, x_{2}\right)=c_{1}\left(x_{1}-\varepsilon\right)^{2}+v\left(x_{1}, x_{2}\right) x_{1} x_{2}+c_{2}\left(x_{2}-\varepsilon\right)^{2}
$$

and consider the problem $P^{\varepsilon}$ which is obtained by substituting $f^{2}$ by $f^{\varepsilon}$. Note that

$$
\begin{aligned}
& D_{x} f^{\varepsilon}(\varepsilon, 0)=\left(0, \varepsilon v(\varepsilon, 0)-2 c_{2} \varepsilon\right), \\
& D_{x} f^{\varepsilon}(0, \varepsilon)=\left(\varepsilon v(0, \varepsilon)-2 c_{1} \varepsilon, 0\right) .
\end{aligned}
$$

Hence, we obtain two different points $(\varepsilon, 0)^{T},(0, \varepsilon)^{T} \in \Sigma^{M}\left(P^{\varepsilon}\right)$ which contradicts that $\bar{x} \in \Sigma^{M S}(\bar{P})$.

Case 2: $\quad \bar{\rho}<0, \bar{\sigma}=0$. Choose $f^{2} \in \mathcal{C}^{\infty}\left(\mathbb{R}^{2}, \mathbb{R}\right)$ such that $f^{1}-f^{2}$ is a small multiple of $x_{2}^{2}$ and that

$$
\frac{\partial^{2} f^{2}(0,0)}{\partial x_{2}^{2}} \neq 0 .
$$


Let $P^{2}$ denote the problem that results from substituting $f^{1}$ by $f^{2}$ in $P^{1}$. Furthermore, for $\varepsilon \in \mathbb{R}$ with

$$
\varepsilon \frac{\partial^{2} f^{2}(0,0)}{\partial x_{2}^{2}}<0
$$

consider the perturbation $f^{\varepsilon}$ of $f^{2}$ given by

$$
f^{\varepsilon}\left(x_{1}, x_{2}\right)=f^{2}\left(x_{1}, x_{2}\right)-\varepsilon x_{2}
$$

and let $P^{\varepsilon}$ be the resulting perturbed problem. Choose $V \in \mathcal{V}(0,0)$ and $W \in$ $\mathcal{W}^{V}\left(P^{2}\right)$ as in Lemma 4.1. We will get a contradiction to $\bar{x} \in \Sigma^{M S}(\bar{P})$ by showing that

$$
\Sigma^{M}\left(P^{\varepsilon}\right) \cap V=\emptyset
$$

for any $\varepsilon$ such that $P^{\varepsilon} \in W$ and that (4.6) holds. Suppose contrarily that there exists $\left(x_{1}^{\varepsilon}, x_{2}^{\varepsilon}\right)^{T} \in \Sigma^{M}\left(P^{\varepsilon}\right) \cap V$ with corresponding Lagrange vector $\left(\rho^{\varepsilon}, \sigma^{\varepsilon}\right)$. By Lemma 4.1, it follows that $\rho^{\varepsilon}<0$ and, by M-stationarity, that $\sigma^{\varepsilon}=0$. Therefore, we have

$$
x_{1}^{\varepsilon}=0, \frac{\partial f^{2}\left(x_{1}^{\varepsilon}, x_{2}^{\varepsilon}\right)}{\partial x_{2}}=\varepsilon .
$$

Now, consider the mapping $G \in \mathcal{C}^{2}\left(\mathbb{R}^{2} \times \mathbb{R}, \mathbb{R}^{2}\right)$ given as

$$
G\left(x_{1}, x_{2}, t\right)=\left(x_{1}, \frac{\partial f^{2}\left(x_{1}, x_{2}\right)}{\partial x_{2}}-t\right)^{T}
$$

with $t \in \mathbb{R}$. Since $D_{x} G(0,0,0)$ is nonsingular, the Implicit Function Theorem yields a uniquely determined and continuously differentiable solution $\hat{x}_{1}(t), \hat{x}_{2}(t)$ for $t$ near the origin with

$$
\begin{array}{r}
G\left(\hat{x}_{1}(t), \hat{x}_{2}(t), t\right)=0, \\
\hat{x}_{1}(0)=\hat{x}_{2}(0)=0 .
\end{array}
$$

By (4.8) and (4.9) we get $x_{2}^{\varepsilon}=\hat{x}_{2}(\varepsilon)$ and a straightforward calculation provides

$$
\frac{\partial \hat{x}_{2}(0)}{\partial t} \frac{\partial^{2} f^{2}(0,0)}{\partial x_{2}^{2}}=1 \text {. }
$$

Hence, by the previous expression and (4.6), we obtain $\frac{\partial \hat{x}_{2}(0)}{\partial t} \varepsilon<0$ and, therefore $x_{2}^{\varepsilon}<0$. This contradicts the feasibility of $\left(x_{1}^{\varepsilon}, x_{2}^{\varepsilon}\right)^{T}$ and completes the proof of this case. Note that the proof of the case $\bar{\rho}=0, \bar{\sigma}<0$ runs analogously.

Obviously, Examples 4.1 and 4.2 correspond in the previous proof to Cases 1 and 2, respectively. In particular, Figs. 1 and 2 illustrate the local changes of the set of M-stationary points after a sufficiently small translation of the graph of $\bar{f}$.

In Theorem 4.1 we presented a necessary condition for the strong stability of an Mstationary point. Our goal is now to state an equivalent algebraic characterization for it. For this, following [27], we consider a relaxed program for $\bar{P}$ at $\bar{x}$ which we denote here by $\bar{P}^{\text {rel }}$. Moreover, we consider the perturbation $P^{\text {rel }}$ of $\bar{P}^{\text {rel }}$ which is a standard nonlinear program given as

$$
P^{\mathrm{rel}}: \min _{x \in M^{\mathrm{rel}}[r, s]} f(x)
$$


with

$$
M^{\mathrm{rel}}[r, s]=\left\{\begin{array}{l|l}
x \in \mathbb{R}^{n} & \begin{array}{l}
r_{m}(x)=0, s_{m}(x) \geq 0, m \in I_{\bar{r}}(\bar{x}) \\
r_{m}(x) \geq 0, s_{m}(x)=0, m \in I_{\bar{s}}(\bar{x}) \\
r_{m}(x) \geq 0, s_{m}(x) \geq 0, m \in I_{\bar{r} \bar{s}}(\bar{x})
\end{array}
\end{array}\right\},
$$

where the functions $f, r$ and $s$ are assumed to be near $\bar{f}, \bar{r}$ and $\bar{s}$, respectively.

Remark 4.1 Let $\bar{P}=\mathcal{P}^{\mathrm{cc}}(\bar{f}, \bar{r}, \bar{s})$ and consider its perturbation $P=\mathcal{P}^{\mathrm{cc}}(f, r, s)$. Note that $P^{\text {rel }}$ does not depend only on the functions that describe $P$, but also on those describing $\bar{P}$ as well as on the point $\bar{x}$.

The following auxiliary result relates locally the M-stationary points of a perturbed MPCC to the stationary points of the corresponding perturbed relaxed program.

Lemma 4.3 Assume that MPCC-LICQ holds at $\bar{x} \in \Sigma^{M}(\bar{P})$. If $I^{\bar{\rho}} \cap I^{\bar{\sigma}}=\emptyset$ and $\bar{\rho}_{m} \geq 0$, $\bar{\sigma}_{m} \geq 0, m \in I_{\bar{r} \bar{s}}(\bar{x})$, then there exist $V \in \mathcal{V}(\bar{x})$ and $W \in \mathcal{W}^{V}(\bar{P})$ such that

$$
\Sigma^{M}(P) \cap V^{\prime}=\Sigma\left(P^{r e l}\right) \cap V^{\prime}
$$

for all $V^{\prime} \subset V$ and all $P \in W$.

Proof Choose $V \in \mathcal{V}(\bar{x})$ and $W \in \mathcal{W}^{V}(\bar{P})$ as in Lemma 4.1. First, we show the inclusion

$$
\Sigma^{M}(P) \cap V \subset \Sigma\left(P^{\text {rel }}\right) \cap V
$$

for all $P \in W$. If $\Sigma^{M}(P) \cap V=\emptyset$, then (4.11) immediately follows. Now, for $P \in W$ choose a point $x \in \Sigma^{M}(P) \cap V$ and note that $x \in M^{\text {rel }}[r, s]$. Moreover, by Lemma 4.1, we obtain $\rho_{m} \geq 0, \sigma_{m} \geq 0, m \in I_{\bar{r} \bar{s}}(\bar{x})$ for $(\rho, \sigma) \in \mathcal{L}^{M}(P, x)$ and, therefore, $x \in \Sigma\left(P^{\text {rel }}\right) \cap V$. Thus, (4.11) holds. Next, we show that

$$
\Sigma\left(P^{\mathrm{rel}}\right) \cap V \subset \Sigma^{M}(P) \cap V
$$

for all $P \in W$. If $\Sigma\left(P^{\text {rel }}\right) \cap V=\emptyset$, then (4.12) immediately follows. Now, choose $x \in$ $\Sigma\left(P^{\text {rel }}\right) \cap V$ and let $(\rho, \sigma) \in \mathcal{L}\left(P^{\text {rel }}, x\right)$ be the uniquely determined Lagrange vector. By continuity and $I^{\bar{\rho}} \cap I^{\bar{\sigma}}=\emptyset$, we get $I^{\rho} \cap I^{\sigma}=\emptyset$. By the latter and using (4.2), we have $x \in M[r, s]$. Since $(\rho, \sigma) \in \mathcal{L}\left(P^{\text {rel }}, x\right)$, it follows that (4.3) holds and, hence, $x \in \Sigma^{M}(P) \cap V$. Consequently, (4.12) holds. By (4.11) and (4.12), it follows that

$$
\Sigma^{M}(P) \cap V=\Sigma\left(P^{\mathrm{rel}}\right) \cap V .
$$

Finally, by (4.13), for $V^{\prime} \subset V$ we obtain

$$
\Sigma^{M}(P) \cap V^{\prime}=\Sigma^{M}(P) \cap V \cap V^{\prime}=\Sigma\left(P^{\text {rel }}\right) \cap V \cap V^{\prime}=\Sigma\left(P^{\text {rel }}\right) \cap V^{\prime},
$$

which completes the proof.

Now, we state an equivalent characterization of strong stability for an M-stationary point.

Theorem 4.2 Assume that MPCC-LICQ holds at $\bar{x} \in \Sigma^{M}(\bar{P})$. Then, the following two conditions are equivalent.

(i) $\bar{x} \in \Sigma^{M S}(\bar{P})$.

(ii) $\bar{x} \in \Sigma^{S}\left(\bar{P}^{r e l}\right)$ and $I^{\bar{\rho}} \cap I^{\bar{\sigma}}=\emptyset$.

Proof (i) $\Rightarrow$ (ii). By Theorem 4.1, we obtain

$$
I^{\bar{\rho}} \cap I^{\bar{\sigma}}=\emptyset, \bar{\rho}_{m} \geq 0, \bar{\sigma}_{m} \geq 0, m \in I_{\bar{r} \bar{s}}(\bar{x}) .
$$


According to Lemma 4.3, there exist $V \in \mathcal{V}(\bar{x})$ and $W \in \mathcal{W}^{V}(\bar{P})$ such that (4.10) holds for all $V^{\prime} \subset V$ and all $P \in W$. By (i) and (4.10), it follows that

$$
\left|\Sigma^{M}(P) \cap V^{\prime}\right|=\left|\Sigma\left(P^{\text {rel }}\right) \cap V^{\prime}\right|=1
$$

and, therefore, $\bar{x} \in \Sigma^{S}\left(\bar{P}^{\text {rel }}\right)$.

(ii) $\Rightarrow$ (i). By the definition of $\bar{P}^{\text {rel }}$ and (ii), we get (4.14). We use again Lemma 4.3 for obtaining (4.15) and, consequently, $\bar{x} \in \Sigma^{M S}(\bar{P})$. This completes the proof.

The main result of this paper is an algebraic characterization for the strong stability of an M-stationary point by taking first and second order information of $(\bar{f}, \bar{r}, \bar{s})$ into account. For this, we need a notation that is analogous to that at the end of Section 3. For each index sets $\bar{J}^{1}, \bar{J}^{2}$ with

$$
\begin{aligned}
& I^{*}(\bar{\rho}) \cup I_{\bar{r}}(\bar{x}) \subset \bar{J}^{1} \subset \bar{I}_{\bar{r}}(\bar{x}), \\
& I^{*}(\bar{\sigma}) \cup I_{\bar{s}}(\bar{x}) \subset \bar{J}^{2} \subset \bar{I}_{\bar{s}}(\bar{x})
\end{aligned}
$$

let $C\left(\bar{J}^{1}, \bar{J}^{2}\right) \in \mathbb{R}^{n \times\left(n-\left|\bar{J}^{1}\right|-\left|\bar{J}^{2}\right|\right)}$ be a matrix whose columns form a basis of the space

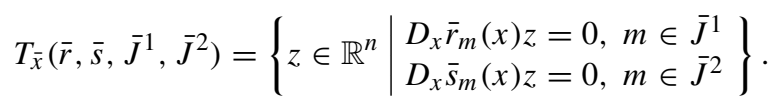

Theorem 4.3 Assume that MPCC-LICQ holds at $\bar{x} \in \Sigma^{M}(\bar{P})$. Then, the following two conditions are equivalent.

(i) $\bar{x} \in \Sigma^{M S}(\bar{P})$.

(ii) $I^{\bar{\rho}} \cap I^{\bar{\sigma}}=\emptyset, \bar{\rho}_{m} \geq 0, \bar{\sigma}_{m} \geq 0, m \in I_{\bar{r} \bar{s}}(\bar{x})$ and

$$
\text { sign } \operatorname{det}\left(C\left(\bar{J}^{1}, \bar{J}^{2}\right)^{T} D_{x}^{2} \overline{\boldsymbol{L}}^{c c}(\bar{x}, \bar{\rho}, \bar{\sigma}) C\left(\bar{J}^{1}, \bar{J}^{2}\right)\right)
$$

is constant and nonvanishing for all $\bar{J}^{1}, \bar{J}^{2}$ satisfying (4.16).

Proof A moment of reflexion shows that after reordering constraints the Lagrange function for $\bar{P}^{\text {rel }}$ is $\overline{\mathbf{L}}^{\text {cc }}$. Moreover, the family of all matrices $C(\bar{J})$ with $\bar{J}$ satisfying (3.2) for $\bar{P}^{\text {rel }}$, and the family of all $C\left(\bar{J}^{1}, \bar{J}^{2}\right)$ with $\bar{J}^{1}, \bar{J}^{2}$ satisfying (4.16) are the same. Thus, by Proposition 3.1 and Theorem 4.2, the desired result follows.

Remark 4.2 Note that condition (ii) in Theorem 4.3 is purely algebraic and does not explicitly use the relaxed program. Furthermore, an alternative proof to Theorem 4.3 can be given by using Theorem 4.1 and [17, Theorem 3.1]. The latter is an algebraic characterization of strong stability for C-stationary points.

Now, we present an example where Theorem 4.3 is applied.

Example 4.3 Let $n=4, \bar{x}=(0,0,0,0)^{T}$ and consider the problem $\bar{P}$ given by

$$
\begin{aligned}
& \min x_{1}^{2}+x_{2}+x_{3}^{2}+x_{4} \\
& \text { s.t. } \\
& \min \left\{x_{1}, x_{2}\right\}=0, \\
& \min \left\{x_{3}, x_{4}\right\}=0,
\end{aligned}
$$


Note that $\bar{\rho}_{1}=\bar{\rho}_{2}=0, \bar{\sigma}_{1}=\bar{\sigma}_{2}=1$ and $D_{x}^{2} \overline{\mathbf{L}}^{\mathrm{cc}}(\bar{x}, \bar{\rho}, \bar{\sigma})=\operatorname{diag}(2,0,2,0)$. Obviously, $I^{\bar{\rho}} \cap I^{\bar{\sigma}}=\emptyset$ and for $\bar{J}^{1}=\bar{J}^{2}=\{1,2\}$ we use Remark 3.1 to get

$$
\text { sign } \operatorname{det}\left(C\left(\bar{J}^{1}, \bar{J}^{2}\right)^{T} D_{x}^{2} \overline{\mathbf{L}}^{\mathrm{cc}}(\bar{x}, \bar{\rho}, \bar{\sigma}) C\left(\bar{J}^{1}, \bar{J}^{2}\right)\right)=1 .
$$

Moreover, the matrices $C\left(\bar{J}^{1}, \bar{J}^{2}\right)$ with $T_{\bar{x}}\left(\bar{r}, \bar{s}, \bar{J}^{1}, \bar{J}^{2}\right) \neq\left\{(0,0,0,0)^{T}\right\}$ are

$$
\left(\begin{array}{l}
1 \\
0 \\
0 \\
0
\end{array}\right),\left(\begin{array}{l}
0 \\
0 \\
1 \\
0
\end{array}\right),\left(\begin{array}{ll}
1 & 0 \\
0 & 0 \\
0 & 1 \\
0 & 0
\end{array}\right)
$$

and a straightforward calculation yields (4.17). Thus, by Theorem 4.3, it follows that $\bar{x} \in$ $\Sigma^{M S}(\bar{P})$. However, note that if we swap $x_{2}$ and $x_{3}$ in the constraints of $\bar{P}$, then $\bar{x}$ is not a strongly stable M-stationary point for the resulting problem.

\section{Final Remarks}

We conclude this paper by presenting some final remarks.

Remark 5.1 Each M-stationary point is also a C-stationary one, but not vice versa; this is nicely illustrated in [17, Example 2.1 and Remark 4.2]. As shown in [16] as a generalization of classical Morse theory, the topological structure of the feasible level set of MPCC is changing if and only if a level is passed that contains a C-stationary point. Therefore, this topological structure (e.g. the number of connected components of the feasible set) is changing when the level of an M-stationary point is passed. Such changes play a crucial role for numerical solution methods via global optimization or homotopy methods.

Remark 5.2 We refer to S-stationarity under MPCC-LICQ which is described by

$$
\bar{\rho}_{m} \geq 0, \bar{\sigma}_{m} \geq 0, m \in I_{\bar{r} \bar{s}}(\bar{x}) .
$$

Obviously, an S-stationary point is also M-stationary but not vice versa. By Theorem 4.2, the strong stability of an M-stationary point $\bar{x}$ implies that $\bar{x}$ is also a strongly stable $\mathrm{S}$ stationary point where the latter is defined analogously. Moreover, if $\bar{x}$ is a local minimizer, then $\bar{x}$ is an S-stationary point. This property is closely related to the discussion in [9] where $\mathrm{S}$-stationarity is considered as the most appropriate stationarity concept for both optimality conditions and sensitivity analysis.

Remark 5.3 Since we assumed that MPCC-LICQ holds at the point $\bar{x} \in \Sigma^{M}(\bar{P})$ under consideration, the corresponding Lagrange vector $(\bar{\rho}, \bar{\sigma})$ is uniquely determined. A moment of reflection shows that, if $\bar{x} \in \Sigma^{M S}(\bar{P})$, then the properties of strong stability (existence, uniqueness and continuous dependence on the perturbation) is not only satisfied for $x$ but also for the pair $(x,(\rho, \sigma))$.

Funding Open access funding provided by University of Bergen (incl Haukeland University Hospital).

Open Access This article is licensed under a Creative Commons Attribution 4.0 International License, which permits use, sharing, adaptation, distribution and reproduction in any medium or format, as long as you give appropriate credit to the original author(s) and the source, provide a link to the Creative Commons licence, and indicate if changes were made. The images or other third party material in this article are included in the article's Creative Commons licence, unless indicated otherwise in a credit line to the material. If material is 
not included in the article's Creative Commons licence and your intended use is not permitted by statutory regulation or exceeds the permitted use, you will need to obtain permission directly from the copyright holder. To view a copy of this licence, visit http://creativecommons.org/licenses/by/4.0/.

\section{References}

1. Červinka, M., Outrata, J.V., Pištěk, M.: On stability of M-stationary points in MPCCs. Set-Valued Var. Anal. 22, 575-595 (2014)

2. Facchinei, F., Jiang, H., Qi, L.: A smoothing method for mathematical programs with equilibrium constraints. Math. Program. 85(1) (1999)

3. Flegel, M.L., Kanzow, C.: On M-stationary points for mathematical programs with equilibrium constraints. J. Math. Anal. Appl. 310(1), 286-302 (2005)

4. Hernandez Escobar, D., Rückmann, J.-J.: MPCC: on necessary conditions for the strong stability of C-stationary points. Optimization 68(2-3), 593-613 (2019)

5. Hernandez Escobar, D., Rückmann, J.-J.: MPCC: strongly stable C-stationary points when the number of active constraints is $n+1$. Optimization 69(5), 1039-1067 (2020)

6. Hernandez Escobar, D., Rückmann, J.-J.: Strongly stable C-stationary points for mathematical programs with complementarity constraints. Math. Program. https://doi.org/10.1007/s10107-020-01553-7 (2020)

7. Hirsch, M.W.: Differential Topology. Springer, New York (1976)

8. Hoheisel, T., Kanzow, C., Schwartz, A.: Theoretical and numerical comparison of relaxation methods for mathematical programs with complementarity constraints. Math. Program. 137, 257-288 (2013)

9. Hu, X., Ralph, D.: A note on sensitivity of value functions of mathematical programs with complementarity constraints. Math. Program. 93(2), 265-279 (2002)

10. Izmailov, A.F.: Mathematical programs with complementarity constraints: regularity, optimality conditions, and sensitivity. Comput. Math. Math. Phys. 44(7), 1145-1164 (2004)

11. Izmailov, A.F., Solodov, M.V.: An active-set newton method for mathematical programs with complementarity constraints. SIAM J. Optim. 19(3), 1003-1027 (2008)

12. Jongen, H.T., Möbert, T., Rückmann, J.-J., Tammer, K.: On inertia and Schur complement in optimization. Linear Algebra Appl. 95, 97-109 (1987)

13. Jongen, H.T., Jonker, P., Twilt, F.: Nonlinear Optimization in Finite Dimensions. Kluwer, Dordrecht (2000)

14. Jongen, H.T., Meer, K., Triesch, E.: Optimization Theory. Springer, New York (2004)

15. Jongen, H.T., Rückmann, J.-J., Shikhman, V.: On stability of the feasible set of a mathematical problem with complementarity problems. SIAM J. Optim. 20(3), 1171-1184 (2009)

16. Jongen, H.T., Rückmann, J.-J., Shikhman, V.: MPCC: critical point theory. SIAM J. Optim. 20(1), 473484 (2009)

17. Jongen, H.T., Shikhman, V., Steffensen, S.: Characterization of strong stability for C-stationary points in MPCC. Math. Program. 132(1), 295-308 (2012)

18. Kanzow, C., Schwartz, A.: Mathematical programs with equilibrium constraints: enhanced Fritz Johnconditions, new constraint qualifications, and improved exact penalty results. SIAM J. Optim. 20(5), 2730-2753 (2010)

19. Kanzow, C., Schwartz, A.: A new regularization method for mathematical programs with complementarity constraints with strong convergence properties. SIAM J. Optim. 23, 770-798 (2016)

20. Klatte, D., Kummer, B.: Strong stability in nonlinear programming revisited. J. Austral. Math. Soc. Ser. B 40(3), 336-352 (1999)

21. Kojima, M.: Strongly stable stationary solutions in nonlinear programs. In: Robinson, S.M. (ed.) Analysis and Computation of Fixed Points, pp. 93-138. Academic Press, New York (1980)

22. Leyffer, S., López-Calva, G., Nocedal, J.: Interior methods for mathematical programs with complementarity constraints. SIAM J. Optim. 17(1), 52-77 (2006)

23. Luo, Z.-Q., Pang, J.-S., Ralph, D.: Mathematical Programs with Equilibrium Constraints. Cambridge University Press, Cambridge (1996)

24. Outrata, J.V.: Optimality conditions for a class of mathematical programs with equilibrium constraints. Math. Oper. Res. 24(3), 627-644 (1999)

25. Robinson, S.M.: Strongly regular generalized equations. Math. Oper. Res. 5(1), 43-62 (1980)

26. Rückmann, J.-J.: On existence and uniqueness of stationary points in semi-infinite optimization. Math. Program. 86(2), 387-415 (1999) 
27. Scheel, H., Scholtes, S.: Mathematical programs with complementarity constraints: stationarity, optimality, and sensitivity. Math. Oper. Res. 25(1), 1-22 (2000)

28. Shikhman, V.: Topological Aspects of Nonsmooth Optimization. Springer, New York (2012)

29. Ye, J.J.: Necessary and sufficient optimality conditions for mathematical programs with equilibrium constraints. J. Math. Anal. Appl. 307(1), 350-369 (2005)

Publisher's Note Springer Nature remains neutral with regard to jurisdictional claims in published maps and institutional affiliations. 